\title{
Campos Potenciais Artificiais e Consenso Aplicados ao Problema de Navegação de Sistemas Multiagente Heterogêneo
}

\author{
George Soon Ho Pereira \\ Instituto Federal de Educação, Ciência e \\ Tecnologia de Roraima (IFRR) \\ Boa Vista, Roraima, Brasil \\ george.pereira@ifrr.edu.br
}

\author{
José Reginaldo Hughes Carvalho \\ Instituto de Computação (ICOMP) \\ Universidade Federal do Amazonas (UFAM) \\ Manaus, Amazonas, Brasil \\ reginaldo@icomp.ufam.edu.br
}

\begin{abstract}
Resumo-Este artigo avalia a eficácia da aplicação combinada da técnica conhecida por Campos Potenciais Artificiais (CPA) com a técnica de Consenso para sistemas multiagente heterogêneos compostos por Veículos Aéreos Não Tripulados (VANT) e Veículos Terrestres Não Tripulados (VTNT). O Consenso é uma técnica muito utilizada para movimentações coordenadas em formação de múltiplos agentes, enquanto que a técnica de CPA é tipicamente usada para a navegação com desvio de obstáculos em sistemas multiagente devido à sua simplicidade e eficiência. Apesar da existência de pesquisas envolvendo o uso de CPA e Consenso em conjunto, nenhuma explora a cooperação de veículos com diferentes capacidades de movimentação. Neste artigo, é apresentada uma arquitetura para sistemas multiagente compostos por VANTs e VTNTs para a execução de tarefas em ambientes de operação de exterior usando a técnica de Consenso para a manutenção da formação, e a técnica de CPA para o desvio de obstáculos. São realizados experimentos em simulações com o objetivo de demonstrar que a utilização de CPA e Consenso em conjunto é uma estratégia efetiva para a navegação em formação de um sistema multiagente composto por VANTs e VTNTs, em ambientes onde há a presença de obstáculos.

Palavras-chave-consenso, campos potenciais artificiais, sistema multiagente, sistemas robóticos heterogêneos, navegação autônoma, comunicação assíncrona
\end{abstract}

\section{INTRODUÇÃo}

Pesquisas aplicadas de Veículos Aéreos Não Tripulados (VANT) e Veículos Terrestres Não Tripulados (VTNT) tem aumentado de forma considerável nos últimos anos. Dentre as principais razões pode-se destacar o aumento da capacidade dos processadores embarcados, aliada à redução de custos dos componentes críticos, tais como placas de processamento, pilotos automáticos, sensores e câmeras, motores, baterias, dentre outros.

Apesar de VANT ou VTNT de diversas classes e preços poderem ser encontrados no mercado, uma nova oportunidade que se vislumbra é o uso combinado de sistemas aéreos e terrestres. Uma consequência imediata é o desenvolvimento de esquemas de navegação conjuntos que promovam as novas aplicações, e sejam ao mesmo tempo robustos e seguros.

Contudo, coordenar VANTs e VTNTs não é uma tarefa trivial [1], pois se tratam de veículos com capacidades de movimentação diferentes. Em especial, a navegação autônoma em formação em ambientes com obstáculos é um dos problemas mais investigados, por ter várias aplicações práticas [2], [3], desde a agricultura de precisão até a exploração espacial.

Uma das abordagens para tratar esse problema é utilizar Consenso em conjunto com Campos Potenciais Artificiais (CPA). A combinação preserva a capacidade dos CPA de reagir ao ambiente enquanto guia para o objetivo, com a capacidade do Consenso de manter os agentes em formação. Apesar de promissor, os trabalhos que utilizam essa combinação, como Gayle [4] e Sun [5], o fazem aplicados a enxames, ou seja, a sistemas multiagente (SMA) homogêneos (todos os agentes são iguais).

Neste artigo, é proposto um modelo para controle de SMA baseado em consenso e CPA para SMAs heterogêneos compostos por VANTs e VTNTs. A contribuição principal desse trabalho é a de mostrar que o uso combinado de consenso e CPA é uma abordagem válida para o problema de navegação autônoma em formação para SMAs compostos por VANTs e VTNTs. Adicionalmente, o trabalho apresenta um conjunto de simulações para diferentes configurações de veículos aéreos e terrestres.

O restante do artigo está organizado como segue. A Seção II descreve os fundamentos matemáticos que são aplicados no trabalho. A Seção III descreve a metodologia dos experimentos de simulação realizados para verificar a aplicação conjunta de CPA e Consenso. A Seção IV apresenta os resultados adquiridos durante as simulações, enquanto que a Seção V conclui o artigo.

\section{DESCRIÇÃo DO MODELO}

O método descrito tem por objetivo utilizar o consenso para manter a formação e campos potenciais artificiais para locomoção do grupo e desvio de obstáculos. O foco principal está na formação, pois em ambientes não estruturados como florestas ou rios, é necessário manter os agentes agrupados por vários fatores como estabelecer uma distância aceitável para que os agentes possam se comunicar e garantir que nenhum agente se perca durante o trajeto. 


\section{A. Arquitetura do SMA}

Cada agente do SMA tem um módulo do ROS (do inglês, Robot Operating System) executando independentemente dos outros agentes, e a única interação entre os agentes do SMA é apenas para troca de informações de posição. Cada módulo do ROS possui três submódulos:

- Navegação: Responsável por mover o agente em direção ao objetivo e evitar colisões com obstáculos;

- Formação: Responsável por manter os agentes em uma formação específica;

- Comando: Responsável por receber os comandos dos dois outros módulos e enviar os comandos apropriados para os atuadores do agente.

\section{B. Módulo de navegação: CPA}

$\mathrm{O}$ módulo de navegação é baseado em CPA, um método de controle para a movimentação de agentes e robôs, composto por campos potenciais atrativos e repulsivos, que geram uma força resultante que fornecem a direção e a velocidade desejada para dirigir o agente até o objetivo e evitar obstáculos.

A ideia básica é determinar um ponto (objetivo) para o qual o agente será atraído, e adicionar outros pontos (obstáculos) que repelem o agente [6]. Cada ponto é convertido em um vetor com direção e velocidade (positiva caso seja atrativo, e negativa caso contrário) em relação à posição atual do agente. Ao somar esses vetores, é dado um vetor resultante que fornece a direção e velocidade que o agente deve adotar [4], [7].

Para representar o campo potencial atrativo, temos:

$$
U_{a t r}=\frac{1}{2} \alpha d^{2}\left(p, p_{o b j}\right)
$$

onde $\alpha$ é uma constante de ganho, e $d\left(p, p_{o b j}\right)$ é a distância entre o agente e o objetivo. A força atrativa é dada pelo gradiente negativo do campo potencial atrativo:

$$
F_{a t r}=-\nabla U_{a t r}
$$

De forma semelhante, para representar o campo potencial repulsivo em um ponto, usamos a função:

$$
U_{\text {rep }}= \begin{cases}\beta\left(\frac{1}{\sqrt{d\left(p, p_{o b s}\right)}}\right), & \text { se } d\left(p, p_{\text {obs }}\right)<i_{\text {obs }} \\ 0, & \text { se } d\left(p, p_{\text {obs }}\right) \geq i_{\text {obs }}\end{cases}
$$

onde $\beta$ é uma constante de ganho, $d\left(p, p_{\text {obs }}\right)$ é a distância entre o agente e o obstáculo, e $i_{o b s}$ é o raio de influência do obstáculo. A força repulsiva é dada pelo gradiente do campo potencial repulsivo:

$$
F_{\text {rep }}=\nabla U_{\text {rep }}
$$

Apesar de ser um método bastante utilizado para a navegação de SMAs, uma limitação inerente aos campos potenciais é o problema de mínimos locais. Existem situações em que $\mathrm{o}$ agente pode estar posicionado em locais onde as forças de atração e repulsão se anulam, fazendo com que o robô fique parado.
O tratamento dos pontos mínimos locais costuma ser feito através de alterações na $F_{r e p}$ ou adição de novas forças na $F_{\text {total }}$ [4], [5], [8], [9], com o objetivo de evitar que as forças se anulem em algum ponto que não seja o ponto objetivo. Para o sistema deste artigo, foi utilizada uma versão modificada da técnica usada por Sun [5], que adiciona à $F_{r e p}$ uma força tangencial aos obstáculos para evitar que o agente se posicione desfavoravelmente.

Considerando um sistema multiagente com $n$ agentes e $j$ obstáculos, podemos definir que o termo de navegação para um agente $i$ é dado pela soma dos campos potenciais atrativos e repulsivos:

$$
F_{\text {total }}(i)=\nabla U_{a t r}-\sum_{j=1}^{n} \nabla U_{r e p}
$$

onde $U_{a t r}$ é dado por:

$$
U_{a t r}=\frac{1}{2} \alpha d^{2}\left(p_{i}, p_{o b j}\right)
$$

sendo $p_{i}$ as coordenadas do agente $i, p_{o b j}$ as coordenadas do objetivo e $d$ a distância. $U_{r e p}$ representa a força de repulsão gerada por cada obstáculo $j$ é dado por:

$$
U_{r e p}=u_{r e p}+R(\theta) u_{r e p}
$$

onde $u_{r e p}$ é dado por:

$$
u_{\text {rep }}= \begin{cases}\beta\left(\frac{1}{\sqrt{d\left(p_{i}, p_{j}\right)}}\right), & \text { se } d\left(p_{i}, p_{j}\right)<i_{o b s} \\ 0, & \text { se } d\left(p_{i}, p_{j}\right) \geq i_{o b s}\end{cases}
$$

sendo $p_{i}$ as coordenadas do agente $i, p_{j}$ as coordenadas do obstáculo $j$, e $i_{\text {obs }}$ a distância segura do obstáculo.

Por fim, $R(\theta)$ é uma matriz de rotação responsável por adicionar uma força tangencial perpendicular à força de repulsão. Como o ângulo de rotação $\theta$ deve ser tangencial à $u_{r e p}$, ele pode assumir os valores $\pi / 2$ para contornar o obstáculo no sentido anti-horário, ou $-\pi / 2$ para contornar o obstáculo no sentido horário. O sentido é definido pela seguinte relação:

$$
\theta= \begin{cases}\pi / 2, & \text { se } \bmod \left(\gamma_{i}-\gamma_{j}, 2 \pi\right) \leq \pi \\ -\pi / 2, & \text { se } \bmod \left(\gamma_{i}-\gamma_{j}, 2 \pi\right)>\pi\end{cases}
$$

onde $\gamma_{i}$ é o ângulo da direção do agente, e $\gamma_{j}$ é o ângulo da linha formada pelo agente e o obstáculo. Tal relação está demonstrada na Figura 1.

\section{Módulo de formação: Consenso}

Consenso é uma técnica de controle de sistemas multiagente que se baseia em fazer que o grupo, por meio da troca de informações, consiga entrar em acordo sobre um determinado valor de interesse, como posição ou velocidade [10]. Neste artigo, o consenso visa manter os agentes em uma formação específica, gerando valores de velocidade ou aceleração baseado na distância de cada agente em relação à posição que ele deveria assumir na formação. Um sistema multiagente pode ser modelado por meio da teoria algébrica dos grafos 


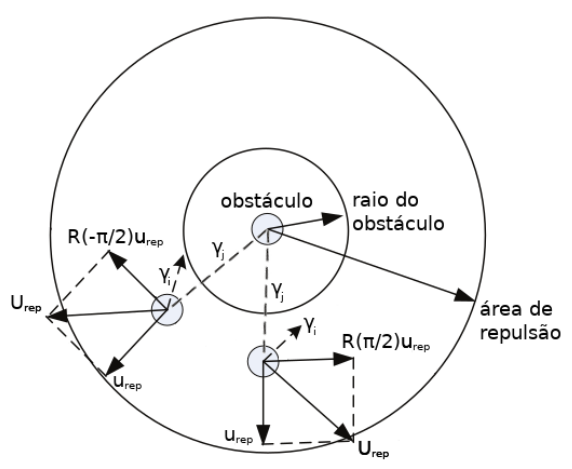

Figura 1. Definição do ângulo $\theta$ para rotação de $u_{r e p}$

considerando que os nós representam os agentes e as arestas, os canais de comunicação entre eles.

$$
\mathcal{G}=(\mathcal{V}, \mathcal{E})
$$

onde, $\mathcal{V}$ é o conjunto dos nós e $\mathcal{E}$ o conjunto das arestas. Para um sistema com $n$ agentes, podemos representar cada um deles por $v_{i}$ e o estado de cada agente, seja ele posição ou outro valor de interesse, por $x_{i} \in \mathbb{R}$, com $i=1, \ldots, n$.

Podemos representar o grafo $\mathcal{G}$ na forma matricial como uma matriz de adjacência, dada pela matriz quadrada $\mathcal{A}=$ $\left[a_{i j}\right]$, cujos elementos são definidos por $a_{i j}=0$ se $i=j$ ou não existir uma aresta conectando $v_{i}$ a $v_{j}$, e $a_{i j}=1$ se existir uma aresta conectando $v_{i}$ a $v_{j}$.

Assim, o agente $v_{i}$ está em consenso com o agente $v_{j}$ apenas quando $x_{i}=x_{j}$, ou seja, somente quando os estados dos dois agentes forem iguais. Assim, podemos dizer que esse sistema está em consenso quando, em um tempo finito e para quaisquer $i$ e $j$, tivermos $x_{i}=x_{j}$.

Para um sistema de tempo contínuo, a lei de controle para um sistema de primeira ordem utilizando consenso costuma ser escrita na forma [11]:

$$
u_{i}(t)=-\sum_{j=1}^{n} a_{i j}\left(x_{i}(t)-x_{j}(t)\right)
$$

onde $u_{i}(t)$ representa a entrada de controle do n-ésimo agente com $i=1, \ldots, n, x_{i}(t)$ e $x_{j}(t)$ representam os estados dos agentes $v_{i}$ e $v_{j}$ respectivamente, $n$ representa o número de agentes, e $a_{i j}$ um elemento da matriz de adjacências. Como em aplicações reais os sistemas multiagente estão sujeitos a atrasos na comunicação entre os agentes e esses atrasos são variantes no tempo, se faz necessário reescrever a lei de controle:

$$
u_{i}(t)=-\sum_{j=1}^{n} a_{i j}\left(x_{i}\left(t-\tau_{i j}(t)\right)-x_{j}\left(t-\tau_{i j}(t)\right)\right)
$$

onde $\tau_{i j}(t)$ representa o atraso entre os agentes.

Partindo dessa formulação, considere um sistema multiagente, composto por $n$ agentes no qual $x_{i} \in R$ representa o estado (qualquer grandeza física como posição, velocidade, temperatura, tensão etc) do agente (nó) $v_{i} \operatorname{com} i=1,2, \ldots, n$. Sabe-se que o agente $v_{i}$ está em consenso com o agente $v_{j}$ se, e somente se, $x_{i}=x_{j}$, ou seja, se o valor dos estados dos agentes $i$ e $j$ forem iguais.

Assim, podemos dizer que tal sistema está em consenso quando, para um tempo suficientemente grande e para quaisquer $i$ e $j$ tivermos $x_{i}=x_{j}$. Matematicamente, por definição, diz-se que ocorreu o consenso em um sistema multiagente se, e somente se, $\lim _{x \rightarrow \infty}\left(x_{i}(t)-x_{j}(t)\right)=0$.

Neste artigo foi usado um sistema multiagente no qual a dinâmica dos $n$ agentes do sistema é igual e de primeira ordem (do tipo integrador simples), como se apresenta a seguir:

$$
\dot{x}_{i}(t)=u_{i}(t)
$$

sendo que $x_{i}(t)$ representa o estado e $u_{i}(t)$ a entrada de controle do i-ésimo agente com $i=1,2, \ldots, n$. Usando como base o modelo definido por Santos [12], podemos definir $u_{i}(t)$ como:

$$
u_{i}(t)=\nabla \sum_{j=1}^{n}\left(p_{i}-p_{j}+k\right)
$$

onde $p_{i}$ são as coordenadas do agente, $p_{j}$ são as coordenadas de outro agente, com $i \neq j$, e $\mathrm{k}$ as distâncias que os agentes devem ter entre si, em cada coordenada.

\section{Módulo de Comando}

Combinando as equações (5) e (13), temos que, para cada agente $i$ :

$$
\dot{x}_{i}(t)=F_{\text {total }}(i)+u_{i}(t)
$$

Este módulo recebe os dados dos módulos de navegação e de formação, e os combina para obter um vetor com a velocidade e direção que o agente deve adotar. Cada agente possui suas especificidades quanto à forma de executar os comandos, e esse módulo se encarrega de adequar os valores adotados para valores apropriados para os atuadores do agente. Apesar do vetor resultante na equação 14 ser igual para todos os agentes, o modo de executar esses valores difere dependendo da dinâmica do agente, e o módulo de comando tem a tarefa de traduzir esses comandos de forma adequada para cada agente.

\section{Metodologia E DescriçÃo dos ExPERIMENTOS}

A fim de verificar se a estratégia de CPA em conjunto com consenso para a navegação de um SMA composto por múltiplos agentes heterogêneos em movimento é um método viável, foram realizados experimentos, conforme descrito a seguir. 


\section{A. Materiais}

Para realizar o experimento, foi utilizado o simulador VREP [13] em conjunto com o software ROS [14]. O V-REP possui um ambiente de simulação com robôs virtuais, que permite simular a física do mundo real no ambiente e nos movimentos dos robôs. Os robôs virtuais utilizados foram o quadrotor simples, e o P3DX, ambos disponíveis na instalação padrão do V-REP. Cada robô está associado a um módulo do ROS, que é executado de forma independente e se comunica com os outros módulos através da interface de rede do próprio ROS.

\section{B. Variáveis independentes}

1) Formações:

- 1 VANT e 1 VTNT (Dupla): Nesta formação, o VANT estaria acima do VTNT, como visto na Figura 2.

- 1 VANT e 3 VTNTs (Quarteto): Nesta formação, os três VTNTs formam um triângulo, e o VANT fica acima de um dos VTNTs, como demonstrado na Figura 3.

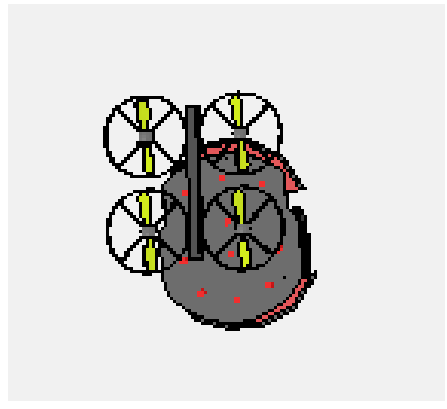

Figura 2. Visão do topo da formação com 1 VANT e 1 VTNT

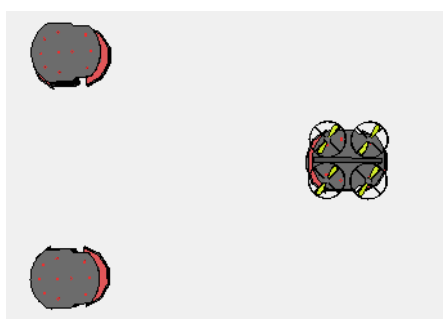

Figura 3. Visão do topo da formação com 1 VANT e 3 VTNT

2) Estratégias de controle:

- Líder-seguidor: um agente virtual é criado com a função de líder, e serve para que o restante dos agentes se baseiem em sua posição para gerar a formação e se movimentar.

3) Cenários:

- Mini floresta: um plano com cinco hastes posicionadas de forma irregular.

- Passagem estreita: dois blocos bloqueiam a passagem pela direita e pela esquerda, deixando apenas uma passagem central.

- Parede: um bloco largo bloqueia a passagem pelo centro, sendo necessário contorná-lo.

\section{Cálculo da Rota de Referência}

Para estabelecer valores de referência para o experimento, cada cenário possui uma rota entre os pontos inicial e final da trajetória, traçada pelo algoritmo $\mathrm{A}^{*}$, que é um algoritmo de busca de caminho, muito utilizado por sua completude, otimalidade e eficiência ótima [15]. Apesar de seu custo computacional elevado que pode inviabilizar o seu uso na prática, ele ainda oferece a melhor solução em muitos casos [16]. Portanto, para oferecer um valor de referência para as variáveis dependentes deste experimento, é feita a comparação da rota do algoritmo $\mathrm{A}^{*}$ com as desenvolvidas pelas formações.

\section{Variáveis dependentes}

- Erro de posicionamento em relação à rota $A^{*}$ : Usando a rota do algoritmo $\mathrm{A}^{*}$ como referência, há a comparação da rota desenvolvida com a solução ótima.

- Erro de posicionamento em relação ao consenso: É verificada a diferença entre a posição de cada agente e a posição em que ele deveria estar na formação, a fim de analisar a manutenção da formação durante o percurso.

\section{E. Metodologia}

No ambiente do V-REP, para cada cenário, inicialmente se obtém a rota do algoritmo $\mathrm{A}^{*}$. A seguir, é executado o experimento 5 vezes a fim de coletar os dados. Para fins de análise são usadas as médias aritméticas dos valores obtidos. Por fim, cada combinação é testada com atrasos incrementais de comunicação (uma vez para cada atraso), até que não se consiga mais atingir o objetivo.

As combinações testadas são:

- Mini floresta, Dupla, Líder-seguidor

- Mini floresta, Quarteto, Líder-seguidor

- Passagem estreita, Dupla, Líder-seguidor

- Passagem estreita, Quarteto, Líder-seguidor

- Parede, Dupla, Líder-seguidor

\section{EXECUÇÃO DOS EXPERIMENTOS}

Como em todos os cenários não houve diferença significativa nas rotas percorridas, apenas uma rota é exibida.

\section{A. Cenário 01: Mini floresta, Dupla, Líder-seguidor}

Os veículos estão dispostos na parte inferior do cenário, e devem atravessar o campo para chegar na parte superior, conforme a Figura 4. Após a execução dos testes, foram obtidos os valores que constam na Tabela I.

Tabela I

VALORES OBTIDOS NOS TESTES E DO ALGORITMO A* NO CENÁRIO 01

\begin{tabular}{|c|l|l|l|l|l|c|}
\hline \multirow{2}{*}{ Veículo } & \multicolumn{7}{|c|}{ Distância Percorrida (metros) } \\
\cline { 2 - 7 } & Teste 1 & Teste 2 & Teste 3 & Teste 4 & Teste 5 & A* \\
\hline P3DX_01 & 11,63 & 11,50 & 11,80 & 11,40 & 11,34 & 9,39 \\
\hline Quad_01 & 11,74 & 11,70 & 11,85 & 11,66 & 11,64 & 8,97 \\
\hline
\end{tabular}

A Figura 5 mostra o trajeto do algoritmo A*, enquanto a Figura 6 mostra um dos trajetos percorridos usando o método proposto. A Figura 7 exibe o erro de posicionamento de cada agente durante o trajeto, em metros. Como o erro de ambos é igual, apenas uma linha é mostrada. 


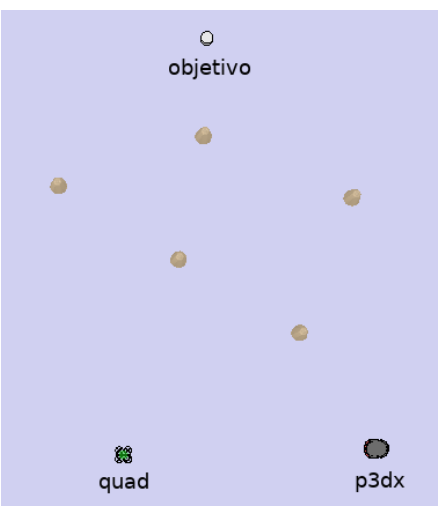

Figura 4. Visão do topo do Cenário 01

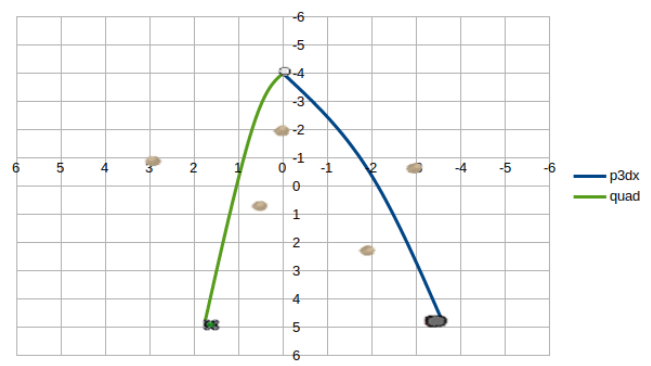

Figura 5. Trajeto usando o algoritmo A*

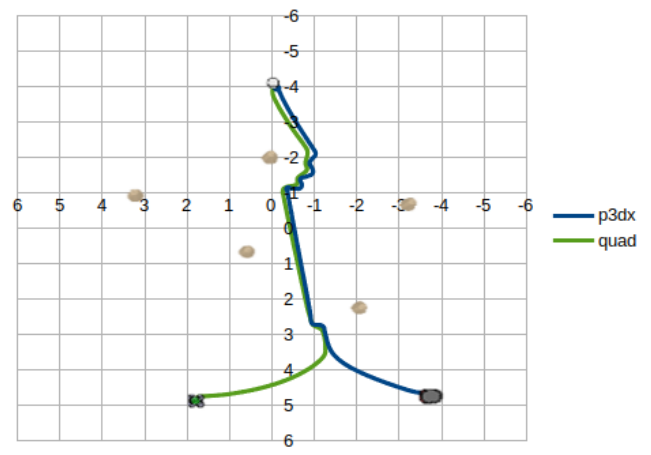

Figura 6. Trajeto usando o método proposto

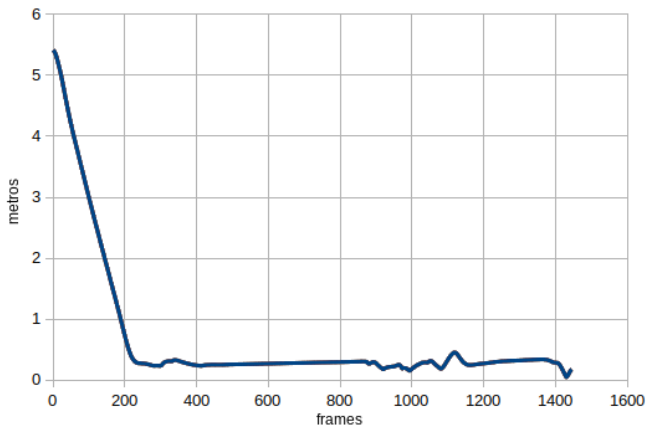

Figura 7. Erro de posicionamento dos agentes usando o método proposto (10 frames $=1$ segundo)

\section{B. Cenário 02: Mini floresta, Quarteto, Líder-seguidor}

Os veículos estão dispostos no topo do cenário, e devem atravessar o campo para chegar na parte inferior, conforme a Figura 8. Após a execução dos testes, foram obtidos os valores que constam na Tabela II.

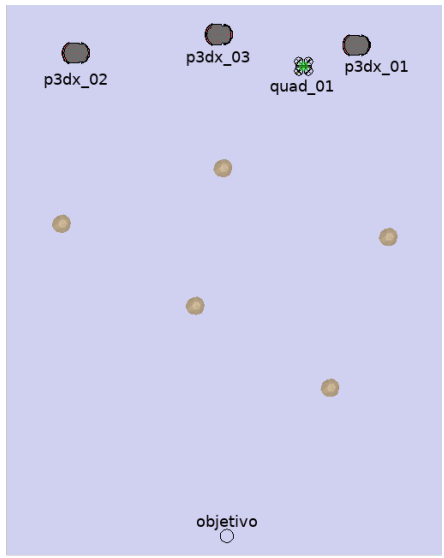

Figura 8. Visão do topo do Cenário 02

A Figura 9 mostra o trajeto do algoritmo $A^{*}$, enquanto a Figura 10 mostra um dos trajetos percorridos usando o método proposto. A Figura 11 exibe o erro de posicionamento de cada agente durante o trajeto, em metros.

Tabela II

VALORES OBTIDOS NOS TESTES E DO ALGORITMO A* NO CENÁRIO 02

\begin{tabular}{|c|l|l|l|l|l|c|}
\hline \multirow{2}{*}{ Veículo } & \multicolumn{7}{|c|}{ Distância Percorrida (metros) } \\
\cline { 2 - 7 } & Teste 1 & Teste 2 & Teste 3 & Teste 4 & Teste 5 & A $^{*}$ \\
\hline P3DX_01 & 9,39 & 9,52 & 9,39 & 9,47 & 9,73 & 9,07 \\
\hline P3DX_02 & 10,73 & 10,91 & 10,88 & 10,60 & 11,02 & 9,94 \\
\hline P3DX_03 & 8,78 & 8,90 & 8,76 & 8,83 & 9,08 & 8,53 \\
\hline Quad_01 & 10,63 & 10,69 & 10,69 & 10,60 & 11,10 & 8,52 \\
\hline
\end{tabular}

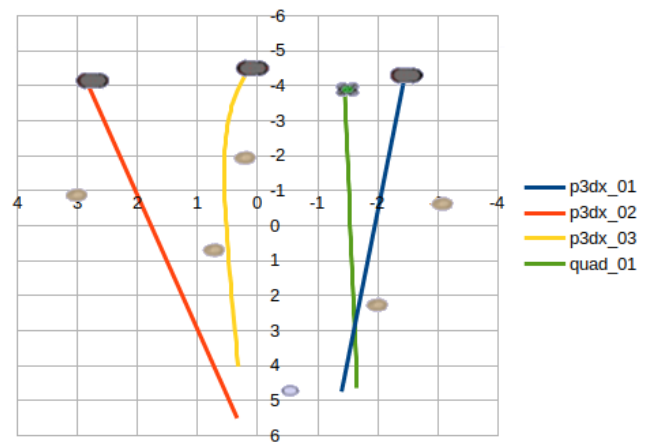

Figura 9. Trajeto usando o algoritmo A*

\section{Cenário 03: Passagem estreita, Dupla, Líder-seguidor}

Os veículos estão dispostos na parte inferior do cenário, e devem atravessar o campo para chegar na parte superior, conforme a Figura 12. Após a execução dos testes, foram obtidos os valores que constam na Tabela III. 


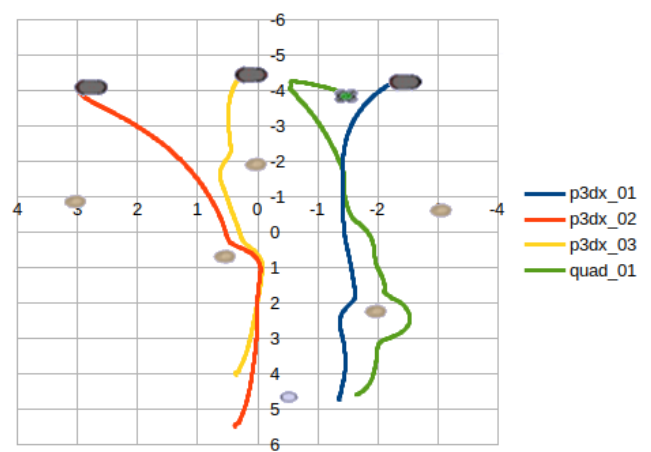

Figura 10. Trajeto usando o método proposto

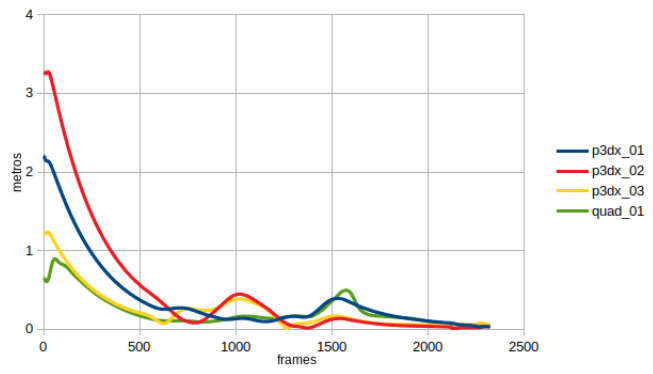

Figura 11. Erro de posicionamento dos agentes usando o método proposto $(10$ frames $=1$ segundo $)$

A Figura 13 mostra o trajeto do algoritmo $A^{*}$, enquanto a Figura 14 mostra um dos trajetos percorridos usando o método proposto. A Figura 15 exibe o erro de posicionamento de cada agente durante o trajeto, em metros. Como o erro de ambos é igual, apenas uma linha é mostrada.

\section{Cenário 04: Passagem estreita, Quarteto, Líder-seguidor}

Os veículos estão dispostos no lado esquerdo do cenário, e devem atravessar o campo para chegar no lado direito, conforme a Figura 16. Após a execução dos testes, foram obtidos os valores que constam na Tabela IV.

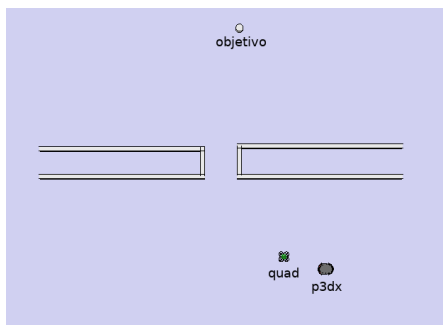

Figura 12. Visão do topo do Cenário 03

Tabela III

VALORES OBTIDOS NOS TESTES E DO ALGORITMO A* NO CENÁRIO 03

\begin{tabular}{|c|l|l|l|l|l|c|}
\hline \multirow{2}{*}{ Veículo } & \multicolumn{7}{|c|}{ Distância Percorrida (metros) } \\
\cline { 2 - 7 } & Teste 1 & Teste 2 & Teste 3 & Teste 4 & Teste 5 & A* \\
\hline P3DX_01 & 12,49 & 12,49 & 12,30 & 15,26 & 12,66 & 8,85 \\
\hline Quad_01 & 12,20 & 12,23 & 12,00 & 12,83 & 12,36 & 9,58 \\
\hline
\end{tabular}

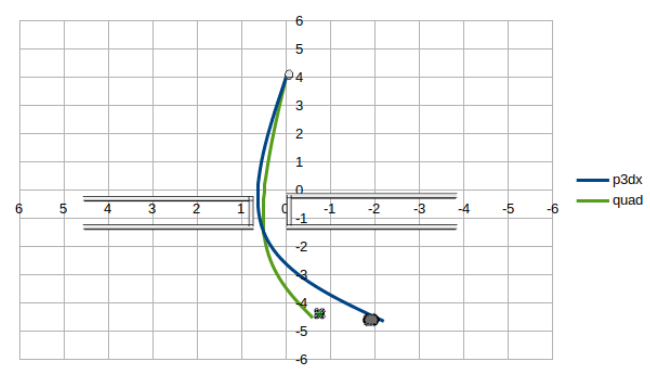

Figura 13. Trajeto usando o algoritmo $\mathrm{A}^{*}$

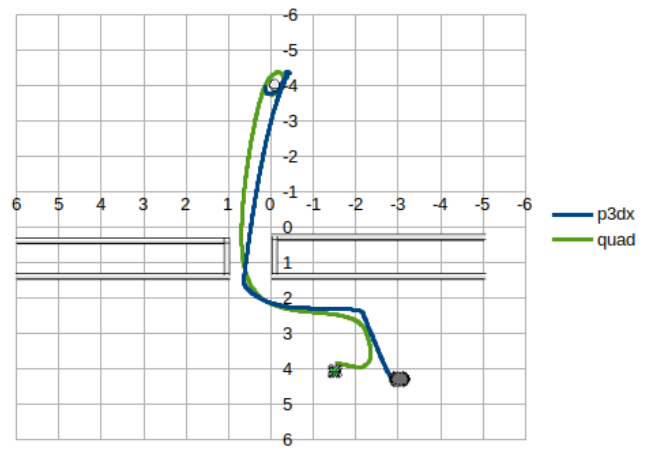

Figura 14. Trajeto usando o método proposto

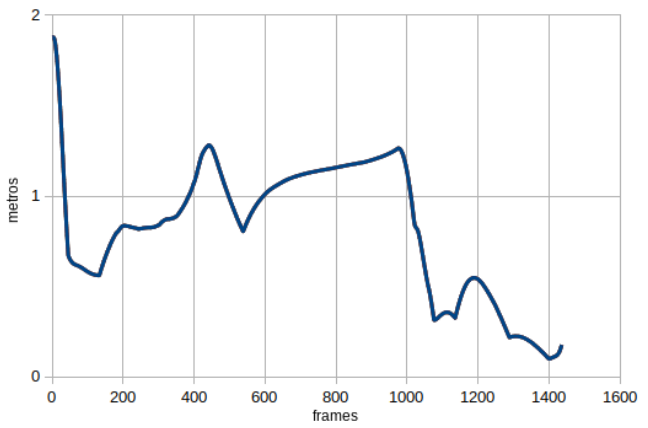

Figura 15. Erro de posicionamento dos agentes usando o método proposto (10 frames $=1$ segundo)

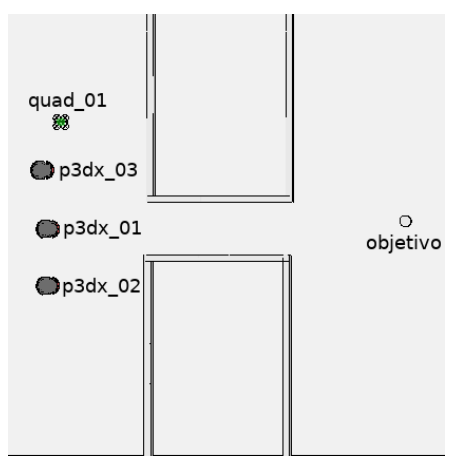

Figura 16. Visão do topo do Cenário 04 
A Figura 17 mostra o trajeto do algoritmo A*, enquanto a Figura 18 mostra um dos trajetos percorridos usando o método proposto. A Figura 19 exibe o erro de posicionamento de cada agente durante o trajeto, em metros.

Tabela IV

VALORES OBTIDOS NOS TESTES E DO ALGORITMO A* NO CENÁRIO 04

\begin{tabular}{|c|l|l|l|l|l|c|}
\hline \multirow{2}{*}{ Veículo } & \multicolumn{7}{|c|}{ Distância Percorrida (metros) } \\
\cline { 2 - 7 } & Teste 1 & Teste 2 & Teste 3 & Teste 4 & Teste 5 & A* \\
\hline P3DX_01 & 9,87 & 9,70 & 9,90 & 9,71 & 9,70 & 9,46 \\
\hline P3DX_02 & 8,80 & 8,77 & 8,83 & 8,79 & 8,80 & 7,92 \\
\hline P3DX_03 & 9,42 & 9,24 & 9,31 & 9,24 & 9,26 & 8,24 \\
\hline Quad_01 & 10,85 & 10,73 & 10,77 & 10,71 & 10,72 & 10,39 \\
\hline
\end{tabular}

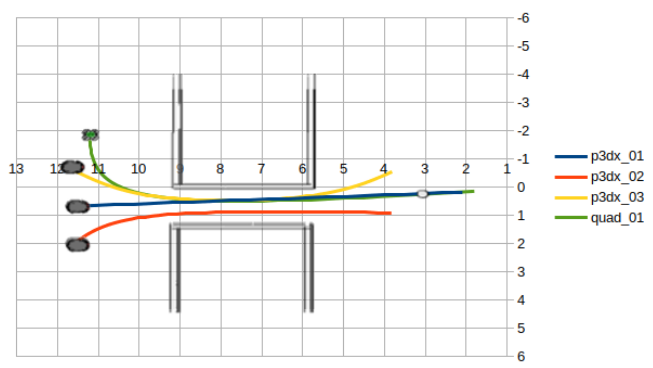

Figura 17. Trajeto usando o algoritmo $\mathrm{A}^{*}$

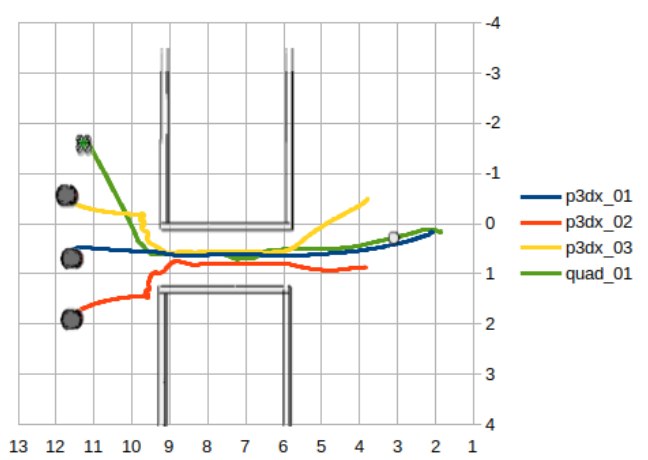

Figura 18. Trajeto usando o método proposto

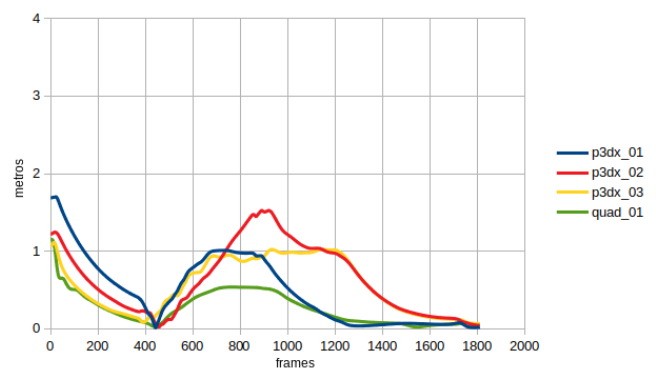

Figura 19. Erro de posicionamento dos agentes usando o método proposto $(10$ frames $=1$ segundo $)$

\section{E. Cenário 05: Parede, Dupla, Líder-seguidor}

Os veículos estão no topo do cenário, e devem atravessar o campo para chegar na parte inferior, conforme a Figura 20.
Após a execução dos testes, foram obtidos os valores que constam na Tabela V.

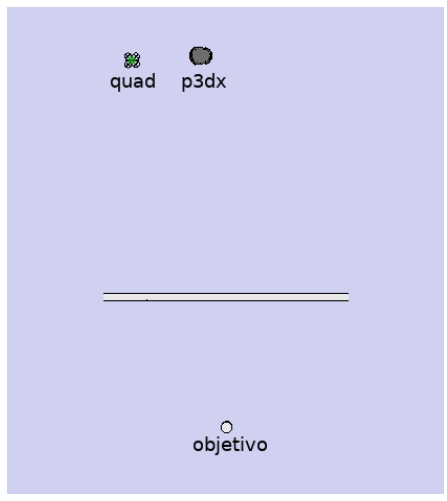

Figura 20. Visão do topo do Cenário 05

Tabela V

VALORES OBTIDOS NOS TESTES E DO ALGORITMO A* NO CENÁRIO 05

\begin{tabular}{|c|c|l|l|l|l|c|}
\hline \multirow{2}{*}{ Veículo } & \multicolumn{7}{|c|}{ Distância Percorrida (metros) } \\
\cline { 2 - 7 } & Teste 1 & Teste 2 & Teste 3 & Teste 4 & Teste 5 & A* \\
\hline P3DX_01 & 17,28 & 17,21 & 17,00 & 17,88 & 17,01 & 10,76 \\
\hline Quad_01 & 18,24 & 18,19 & 18,26 & 18,36 & 17,92 & 10,12 \\
\hline
\end{tabular}

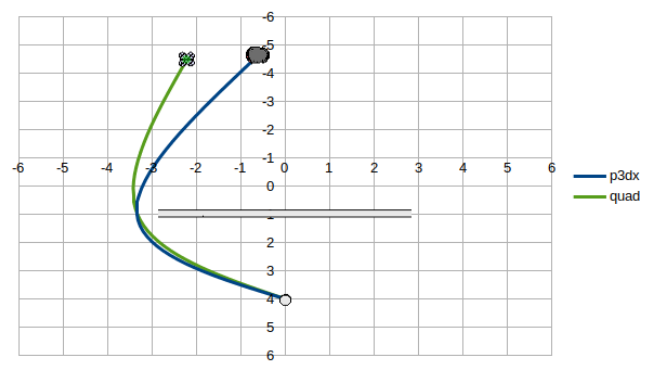

Figura 21. Trajeto usando o algoritmo $\mathrm{A}^{*}$

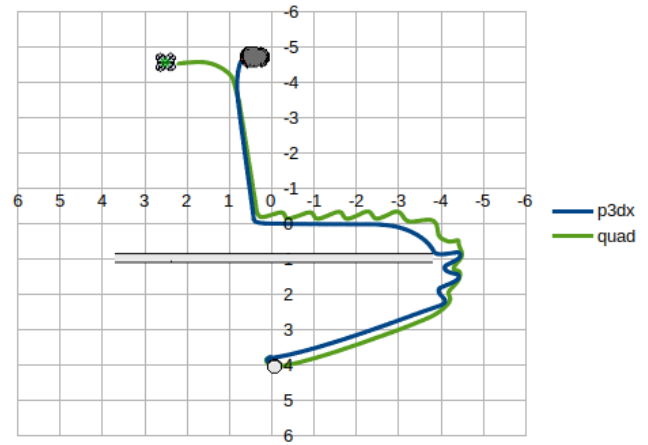

Figura 22. Trajeto usando o método proposto

A Figura 21 mostra o trajeto do algoritmo A*, enquanto a Figura 22 mostra um dos trajetos percorridos usando o método proposto. A Figura 23 exibe o erro de posicionamento de cada agente durante o trajeto, em metros. Como o erro de ambos é igual, apenas uma linha é mostrada. 


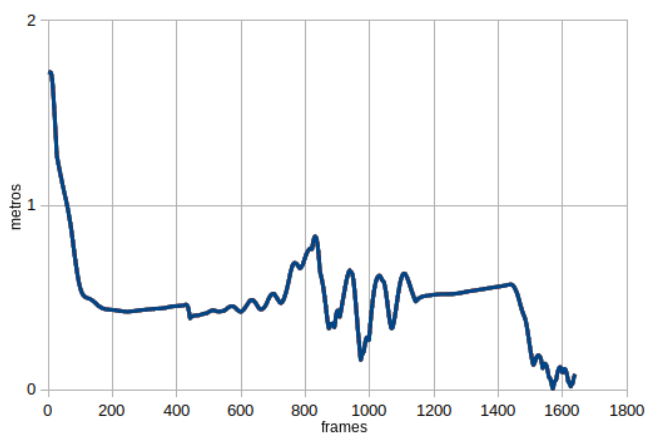

Figura 23. Erro de posicionamento dos agentes usando o método proposto $(10$ frames $=1$ segundo $)$

\section{F. Análise dos resultados}

Analisando os dados obtidoss, vemos a porcentagem de distância percorrida em relação ao algoritmo $\mathrm{A}^{*}$, conforme Tabela VI. Em geral, o aumento do caminho percorrido varia de $2,49 \%$ a $81,45 \%$, com uma média de $24,56 \%$.

Agrupando os dados pelo tipo de formação, temos:

- Dupla: entre $20,71 \%$ e $81,45 \%$, média $44,94 \%$

- Quarteto: entre $2,49 \%$ e $30,34 \%$, média $9,28 \%$

De modo semelhante, agrupando por cenários:

- Mini floresta: entre 3,99\% e 30,67\%, média $16,18 \%$

- Passagem estreita: entre 3,32\% e 47,24\%, média $17,74 \%$

- Parede: entre $60,49 \%$ e $79,81 \%$, média $70,15 \%$

Por fim, agrupando por tipo de agente temos:

- VTNT: mínimo 3,32\%, máximo 60,49\%, média 19,46\%

- VANT: mínimo $3,52 \%$, máximo $79,81 \%$, média $33,75 \%$

Tabela VI

VISÃO GERAL DOS RESULTADOS

\begin{tabular}{|c|c|c|c|}
\hline Cenário & Agentes & $\begin{array}{l}\text { Desempenho } \\
\text { médio }\end{array}$ & $\begin{array}{c}\text { Atraso } \\
\text { máximo }\end{array}$ \\
\hline $\begin{array}{l}\text { Mini floresta } \\
\text { Líder-seguidor }\end{array}$ & $\begin{array}{l}1 \text { VANT } \\
1 \text { VTNT }\end{array}$ & $+26,73 \%$ & $700 \mathrm{~ms}$ \\
\hline $\begin{array}{l}\text { Mini floresta } \\
\text { Líder-seguidor }\end{array}$ & $\begin{array}{l}1 \text { VANT } \\
3 \text { VTNT }\end{array}$ & $\%$ & $500 \mathrm{~ms}$ \\
\hline $\begin{array}{l}\text { Passagem estreita } \\
\text { Líder-seguidor }\end{array}$ & $\begin{array}{l}1 \text { VANT } \\
1 \text { VTNT }\end{array}$ & 10 & $500 \mathrm{~ms}$ \\
\hline $\begin{array}{l}\text { Passagem estreita } \\
\text { Líder-seguidor }\end{array}$ & $\begin{array}{l}1 \text { VANT } \\
3 \text { VTNT }\end{array}$ & $+7,64 \%$ & $400 \mathrm{~ms}$ \\
\hline $\begin{array}{c}\text { Parede } \\
\text { Líder-seguidor }\end{array}$ & $\begin{array}{l}1 \text { VANT } \\
1 \text { VTNT }\end{array}$ & $+70,15 \%$ & 500 \\
\hline
\end{tabular}

\section{Conclusões}

Este artigo apresentou um método para controle de sistemas multiagente heterogêneos em formação, utilizando consenso para o controle de formação juntamente com campos potenciais artificiais para navegação.

Os resultados obtidos demonstram que o método proposto consegue navegar por um ambiente e manter a formação do sistema multiagente de forma eficiente, apresentando resultados satisfatórios para viabilizar o método na vida real.

No entanto, uma limitação deste método está na velocidade máxima em que os agentes podem se mover com segurança.
Em média, a velocidade segura quando se trabalha sem atraso é de $1,5 \mathrm{~m} / \mathrm{s}$, enquanto que com atraso, esse valor cai para 1 $\mathrm{m} / \mathrm{s}$. Outro ponto é o do tamanho do SMA. De acordo com os dados da Tabela VI, pode se observar que o desempenho é melhor quando há mais agentes presentes, o que sugere que o método seja mais eficiente em grupos maiores.

\section{ACKNOWLEDGMENT}

O presente trabalho foi realizado com apoio da Coordenação de Aperfeiçoamento de Pessoal de Nível Superior - Brasil (CAPES) - Código de Financiamento 001.

\section{REFERENNCIAS}

[1] J. Chen, X. Zhang, B. Xin, and H. Fang, "Coordination Between Unmanned Aerial and Ground Vehicles: A Taxonomy and Optimization Perspective," IEEE Transactions on Cybernetics, vol. 46, no. 4, pp. 959972, 2016.

[2] H. Duan and S. Liu, "Unmanned air/ground vehicles heterogeneous cooperative techniques: Current status and prospects," Science China Technological Sciences, vol. 53, no. 5, pp. 1349-1355, 2010.

[3] J. Machado, M. d. F. R. Padilha, F. P. Lira, J. G. De Oliveira, R. S. Silva, and M. B. C. Caetano, "Precision agriculture and opening new frontiers in Brazil," Revista Geama, vol. 4, no. 1, pp. 49-53, 2018.

[4] R. Gayle, W. Moss, M. C. Lin, and D. Manocha, "Multi-robot coordination using generalized social potential fields," 2009 IEEE International Conference on Robotics and Automation, pp. 106-113, 200901. [Online]. Available: http://ieeexplore.ieee.org/document/5152765/

[5] Z. Sun, Y. Xia, and X. Na, "Consensus-based formation control with dynamic role assignment and obstacle avoidance," IMA Journal of Mathematical Control and Information, p. dnv047, 2015. [Online]. Available: https://academic.oup.com/imamci/articlelookup/doi/10.1093/imamci/dnv047

[6] M. Goodrich, "Potential Fields Tutorial," Class Notes, pp. 1-9, 2002. [Online]. Available: http://students.cs.byu.edu/ cs470ta/goodrich/fall2004/lectures/Pfields.pdf

[7] H.-M. Eduardo, "Collision Avoidance in Formation Control Using Discontinuous Vector Fields," IFAC Proceedings Volumes, vol. 46, no. 23, pp. 797-802, 2013. [Online]. Available: http://www.sciencedirect.com/science/article/pii/S1474667016317578

[8] H. H. Triharminto, O. Wahyunggoro, T. B. Adji, A. I. Cahyadi, and I. Ardiyanto, "A novel of repulsive function on artificial potential field for robot path planning," International Journal of Electrical and Computer Engineering, vol. 6, no. 6, pp. 3262-3275, 2016.

[9] X. Liu, S. S. Ge, and C. H. Goh, "Formation Potential Field for Trajectory Tracking Control of Multi-Agents in Constrained Space," International Journal of Control, vol. 90, no. 10, pp. 2137-2151, 2017. [Online]. Available: http://dx.doi.org/10.1080/00207179.2016.1237044

[10] H. J. Savino, F. O. Souza, and L. C. A. Pimenta, "Consensus with guaranteed convergence rate of high-order integrator agents in the presence of time-varying delays," International Journal of Systems Science, vol. 47, no. 10, pp. 2475-2486, 2016.

[11] W. Ren and R. W. Beard, Distributed Consensus in Multi-vehicle Cooperative Control, ser. Communications and Control Engineering. London: Springer London, 2008, vol. 132. [Online]. Available: http://link.springer.com/10.1007/978-1-84800-015-5

[12] C. R. P. dos Santos Junior, J. R. H. Carvalho, and F. O. Souza, "Consenso em sistemas multi-agentes heterogêneos sujeitos a retardos variantes nas acoes de controle," XXI Congresso Brasileiro de Autoática - CBA 2016, 2016.

[13] E. Rohmer, S. Singh, and M. Freese, "V-rep: A versatile and scalable robot simulation framework," IEEE/RSJ International Conference on Intelligent Robots and Systems, pp. 1321-1326, 112013.

[14] M. Quigley, K. Conley, B. Gerkey, J. Faust, T. Foote, J. Leibs, R. Wheeler, and A. Ng, "Ros: an open-source robot operating system," vol. 3, 012009.

[15] S. J. Russell and P. Norvig, Artificial intelligence a modern approach. Boston: Pearson, 2018.

[16] W. Zeng and R. L. Church, "Finding shortest paths on real road networks: the case for a*," International Journal of Geographical Information Science, vol. 23, no. 4, pp. 531-543, 2009. [Online]. Available: https://doi.org/10.1080/13658810801949850 